\title{
Transgenic Reporter Mice With Promoter Region of Murine LRAT Specifically Marks Lens and Meiosis Spermatocytes
}

\author{
D. PRUKOVA ${ }^{1}$, Z. ILENINOVA ${ }^{1}$, B. ANTOSOVA ${ }^{2}$, P. KASPAREK ${ }^{1}$, M. GREGOR ${ }^{1}$, \\ R. SEDLACEK ${ }^{1}$ \\ ${ }^{1}$ Laboratory of Transgenic Models of Diseases, Institute of Molecular Genetics, Czech Academy \\ of Sciences, Prague, Czech Republic, ${ }^{2}$ Laboratory of Transcriptional Regulation, Institute \\ of Molecular Genetics, Czech Academy of Sciences, Prague, Czech Republic
}

Received January 8, 2014

Accepted July 21, 2014

On-line October 15, 2014

\begin{abstract}
Summary
Lecithin:retinol acyltransferase (LRAT) is the major enzyme responsible for retinol esterification in the mammalian body. LRAT exhibits specific activity in the cells with active retinol metabolism where it converts retinols into retinyl esters, which represents the major storage form of retinol. Besides hepatic stellate cells in the liver, LRAT appears to have a key physiologic role in several other tissues. In this study, we generated a transgenic reporter mouse expressing green fluorescence protein (EGFP) under the control of region containing -1166 bps from promoter upstream from the putative transcriptional start site and 262 bps downstream of this start. Transgenic reporter mice exhibited specific expression in eyes and testes. In eyes, expression of EGFP-reporter is found in lens and lens epithelium and fibers from embryo to adulthood. In testes, LRAT-EGFP reporter is expressed both in Sertoli and in spermatocytes marking initiation of spermatogenesis in prepubertal mice. Our data show that the examined LRAT regulatory region is sufficient to achieve strong and selective expression in the eye and testes but not in liver and other organs.
\end{abstract}

\section{Key words}

LRAT • Transgenic mouse - Retinol • Testis - Spermatocyte • Sertoli cell • Eye • Lens

\section{Corresponding authors}

R. Sedlacek, Laboratory of Transgenic Models of Diseases, Institute of Molecular Genetics of the CAS, v. v. i., Videnska 1083, 14220 Prague 4, Czech Republic.

E-mail: radislav.sedlacek@img.cas.cz and

M. Gregor, Laboratory of Transgenic Models of Diseases, Institute of Molecular Genetics of the CAS, v. v. i., Videnska 1083, 14220 Prague 4, Czech Republic.

E-mail: martin.gregor@img.cas.cz

\section{Introduction}

Retinoids plays key role in many essential physiologic functions including growth and development, reproduction, epidermal barrier functions, immune system, and vision. Vitamin A, all-trans-retinol, is delivered into metabolism from dietary uptake in the form of retinols or pro-vitamin carotenoids. Retinols are metabolized into active derivatives including 11-cisretinal and all-trans-retinoic acid, which is essential for transcriptional gene regulation as well as vision. Retinols are also converted into retinyl esters representing the major storage form of retinol in organs such as liver, prostate, breast, kidney, and testes (D'Ambrosio et al. 2011, Guo et al. 2002, Hogarth and Griswold 2010). The liver is the most important organ for storage of retinol that is transported to other tissues with retinoid metabolism (Paik et al. 2004).

Two enzymes, lecithin:retinol acyltransferase (LRAT) and acyl CoA:retinol acyltransferase (ARAT) catalyze retinyl ester synthesis although LRAT appears to be the main enzyme for retinol esterification in the majority of tissues. LRAT acts together with STRA6 and the retinol binding protein (RBP4) (Kawaguchi et al. 2007, O'Byrne and Blaner 2013) and its activity was 
found in various tissues with active metabolism of retinol including the liver, lung, eye, testis, small intestine, skin, mammary gland, and prostate epithelium (reviewed in (Liu et al. 2008)).

LRAT plays a key role in the liver where it is coresponsible for retinyl ester storage (Liu et al. 2008, O'Byrne and Blaner 2013) although this enzyme is crucial also for physiology of other organs such as testis and eyes (Batten et al. 2004). In testis, LRAT and retinol are essential in the process of spermatogenesis (Hogarth and Griswold 2010, Li et al. 2011). LRAT is expressed within Sertoli cells and maintain an optimal level of retinoid for development of spermatozoa (Shingleton et al. 1989). Lrat-deficient mice maintained on vitamin A-deficient diet resulted in meiotic failure and accumulation of undifferentiated spermatogonia in prepubertal mouse testis (Li et al. 2011).

In the eyes, LRAT is present in retinal pigment epithelial cells (RPE) and contributes to maintaining visual cycle by incorporating all-trans-retinol into retina and adjusting its concentration in the RPE (Batten et al. 2004, Saari et al. 1993). The main source of retinol is derived from dietary intake of both retinol and provitamin A carotenoids. Retinols are esterified by LRAT to all-trans-retinyl esters that are stored in retinosomes (Perusek and Maeda 2013). In visual function, 11-cisretinal functions as the chromophore for the pigments present in the rod and cone photoreceptor cells (McBee et al. 2001, Wald 1968). Mice lacking LRAT in RPE showed strong reduction in their ability to synthesize alltrans retinyl esters and a reduced light response in ERG recordings (Ruiz et al. 2007).

To better understand the regulation and expression of LRAT, we generated transgenic reporter C57BL/6N-Tg(LRAT-EGFP) mice expressing EGFP under the control of 5'-untranslated region from -1166 from putative transcriptional start site and $262 \mathrm{bps}$ downstream sequence. The results suggest that the selected part of the regulatory 5'-untranslated region specifically drives expression of green fluorescence protein (EGFP) in eyes and testis although the liver and other organs do not show any reporter expression. In eyes, expression of EGFP-reporter is found in lens, lens epithelium and fibers. In testes, the reporter is expressed both in Sertoli and in spermatocytes marking initiation of spermatogenesis in prepubertal mice.

\section{Material and Methods}

Transgenic construct and generation of C57BL/6N$\operatorname{Tg}($ LRAT-EGFP) transgenic mice

The mouse LRAT promoter fragment $-1166 /+262$ (numbering based on GenBank entry NM_023624, relative to the transcriptional start site), synthesized by GenScript, was excised by XbaI and BspHI from the pUC57 plasmid and inserted into the NheI and NcoI, respectively, in Sleeping Beauty (SB)based vector, pT2/Venus was generously provided by Lajos Mátés (Mates et al. 2009). The resulting construct comprised LRAT promoter, EGFP, chimeric intron (from pRL/TK plasmid (Promega)) and SV40 polyA signal between transposon inverted terminal repeats. To generate transgenic mice, both construct and SB transposase mRNA were used for pronuclear microinjection into E0.5 embryos derived from C57BL/6N strain. Offspring were weaned, genomic DNA for genotyping was isolated from tail snips and 17 founders with the LRAT-EGFP transgene were identified. The F0 generation animals were bred further onto C57BL/6N background. All animal work was performed according to approval by the Animal Care Committee of the Institute of Molecular Genetics in conformity with institutional and national guidelines.

\section{Genotyping}

Genomic DNA was obtained by lysing tail snips in DNA lysis buffer (100 mM Tris- $\mathrm{HCl}$ pH 8.5, $200 \mathrm{mM}$ EDTA, $5 \mathrm{mM} \mathrm{NaCl}, 0.2 \% \mathrm{SDS}$, Proteinase $\mathrm{K}$ at $240 \mu \mathrm{g} / \mathrm{ml}$ ) and incubated at $55^{\circ} \mathrm{C}$ overnight. Samples were extracted with phenol/chloroform, the water phase precipitated by an equal volume of $96 \%$ ethanol, rinsed in $70 \%$ ethanol and resuspended in double-distilled water. Genotyping PCR reaction with DreamTaq Green DNA Polymerase (Thermo Scientific, Waltham, MA) was run as following: $95^{\circ} \mathrm{C} 2 \mathrm{~min} 30 \mathrm{~s} 35$ cycles, $95^{\circ} \mathrm{C}$ $30 \mathrm{~s}, 59.5^{\circ} \mathrm{C} 30 \mathrm{~s}, 72^{\circ} \mathrm{C} 1 \mathrm{~min}, 72^{\circ} \mathrm{C} 7 \mathrm{~min}$. To detect transgenic construct following pairs of forward $(\mathrm{F})$ and reverse $(\mathrm{R})$ primers were used:

LRAT:

F 5'-ATCTCTCCTACGCTGGCTGT-3', R 5'-TGCTCAGGTAGTGGTTGTCG-3'; EGFP:

F 5'-AGTCGTTGTGTGAAGCGAAG-3', R 5'-TGCTCAGGTAGTGGTTGTCG-3'. 


\section{RNA extraction and analysis}

Mouse tissues, were homogenized using TissueLyzer II in TRI-Reagent (Sigma-Aldrich, St. Louis, MO) and RNA was subsequently isolated according to the manufacturer's instructions. RNA pellets were resuspended in double-distilled water; concentration was determined by Nanodrop (Thermo Scientific). Residuals genomic DNA were removed using $1 \mathrm{U}$ of DNAse I (Thermo Scientific)/1 $\mu \mathrm{g}$ RNA by $15 \mathrm{~min}$ incubation at $37^{\circ} \mathrm{C}$, DNase was than inactivated for $5 \mathrm{~min}$ at $70^{\circ} \mathrm{C}$. $1 \mu \mathrm{g}$ of RNA was reverse transcribed to cDNA by $\mathrm{M}$ MLV Reverse Transcriptase (Promega) using oligo (dT) primers. RT-PCR was performed in a $10 \mu \mathrm{l}$ reaction mixture consisting of PCR buffer (Thermo Scientific), $2 \mathrm{mM} \mathrm{MgCl}, 0.2 \mathrm{mM}$ of each dNTP (Sigma-Aldrich), $0.2 \mathrm{mM}$ of each primer, and 1.5 U TaqDNA polymerase (Thermo Scientific). To detect reporter expression the same EGFP primers as above were used. The glyceraldehyde-3-phosphate dehydrogenase (GAPDH) gene was used as an internal control (primers:

F 5'-AATCCCATCACCATCTTCCA-3', R 5'-TGGACTCCACGACGTACTCA-3').

PCR was performed using: $95^{\circ} \mathrm{C} 3$ min 41 cycles, $95^{\circ} \mathrm{C}$ $20 \mathrm{~s}, 60^{\circ} \mathrm{C} 30 \mathrm{~s}, 72{ }^{\circ} \mathrm{C} 30 \mathrm{~s}, 72{ }^{\circ} \mathrm{C} 5 \mathrm{~min}$. Amplified PCR products were run on $1.5 \%$ agarose gels containing $0.5 \mathrm{~g} / \mathrm{ml}$ ethidium bromide.

\section{Western blot analysis}

Mouse tissues, frozen on dry ice, were homogenized using TissueLyzer II in Triton lysis buffer (50 mM Tris pH 7.5, $1 \%$ Triton X-100, $150 \mathrm{mM} \mathrm{NaCl,}$ $1 \%$ DOC, $0.1 \%$ SDS) supplemented with Complete Protease Inhibitor Cocktail (Roche). Protein concentration was measured with BCA (Pierce, Thermo Scientific). The amount of $50 \mu \mathrm{g}$ of tissue homogenate was separated on $12.5 \%$ SDS-PAGE, transferred onto the nitrocellulose membrane (Sigma-Aldrich), blocked with $3 \%$ bovine serum albumin (BSA) in Tris-buffered saline buffer supplemented with $0.1 \%$ Tween (PBST) and incubated overnight at $4{ }^{\circ} \mathrm{C}$ with primary antibody anti-EGFP (sc-9996, Santa Cruz Biotechnology, Texas) or anti-GAPDH (G8795, Sigma-Aldrich) diluted in $2 \%$ non-fat dry milk/PBST. After washing the blots were incubated with horseradish peroxidase-conjugated secondary antibodies (Jackson Laboratories). Immunoreactive proteins were detected with the chemiluminescence system ECL (Rockland Immunochemical, Gilbertsville, PA) and documented by
LAS 3000 (Fuji Photo Film, Tokyo).

\section{Embedding and sectioning}

Dissected mouse tissues were fixed in $4 \%$ paraformaldehyde (PFA) in phosphate-buffered saline (PBS) several hours at $4{ }^{\circ} \mathrm{C}$, then washed three times in PBS at $4{ }^{\circ} \mathrm{C}$ for $5 \mathrm{~min}$ each. For cryo-embedding (Johnson et al. 2007), they were transferred to a solution containing $30 \%$ sucrose in PBS and rocked overnight at $4{ }^{\circ} \mathrm{C}$ then embedded in optimal cutting temperature compound (OCT, Tissue-Tek, Sakura Finetek, Alphen aan den Rijn) in cryosection moulds (Sakura Finetek) and stored at $-80^{\circ} \mathrm{C}$. Embedded tissues were cut in serial sections of $10 \mu \mathrm{m}$ thickness using a Leica cryostat, mounted on Superfrost Plus slides (Millian, Vernier), and stored at $-20^{\circ} \mathrm{C}$ until further use.

\section{Immunostaining}

For immunostaining, all procedures were carried out in a humidified chamber. Slides were blocked for $1 \mathrm{~h}$ in blocking buffer (PBS plus $0.1 \%$ Tween 20 or PBT containing $10 \%$ heat-inactivated sheep serum). Sections were incubated overnight at $4{ }^{\circ} \mathrm{C}$ with primary antibody diluted in blocking buffer. Next day, samples were washed $3 \times 5 \mathrm{~min}$ in PBT and blocked in blocking buffer for $30 \mathrm{~min}$ followed by a $2 \mathrm{~h}$ incubation at room temperature with appropriate secondary antibody diluted in blocking buffer containing 4',6-diamidino-2phenylindole dihydrochloride (DAPI; D9542, SigmaAldrich, $1 \mu \mathrm{g} / \mathrm{ml}$ in PBS). Finally, sections were washed $3 \times 5$ min in PBT and mounted in Mowiol (4-88, SigmaAldrich, used according to http://www.niaid.nih.gov/ LabsAndResources/labs/aboutlabs/rtb/biologicalImaging/ protocols/Pages/mowiolPreparation.aspx). Primary antibodies used in this study were: 1:500 anti-EGFP (A6455, Invitrogen, Life Technologies, Carlsbad, CA), 1:500 rabbit anti-DDX4 (ab13840, Abcam, Cambridge), 1:400 mouse monoclonal anti-SCP3 (sc-74569, Santa Cruz Biotechnology). Secondary antibodies were: 1:500 anti-rabbit IgG A594 (A-11037; Invitrogen). For colocalization, the second primary antibodies were conjugated with A488 using the Zynon kit (Invitrogen). Stained sections were examined using a DMI6000 Leica confocal microscope equipped with HCX PL APO 20x/0.7 imm corr. Lbd. BL, HCX PL APO 40x/1.25-0.75 Oil CS UV lenses. Images were acquired with a Leica Camera DFC350FX R2, a high sensitivity monochrome digital camera with 1.4 megapixel resolution. 
A

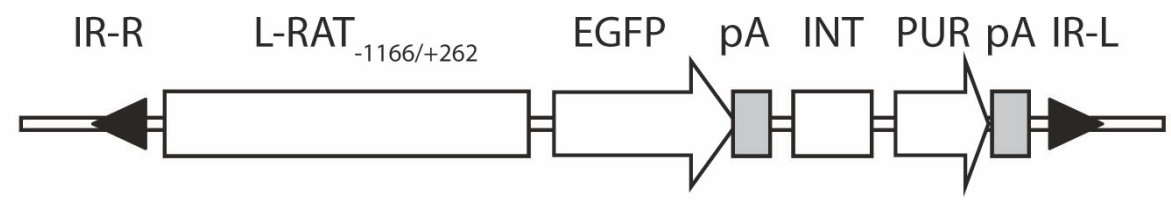

B

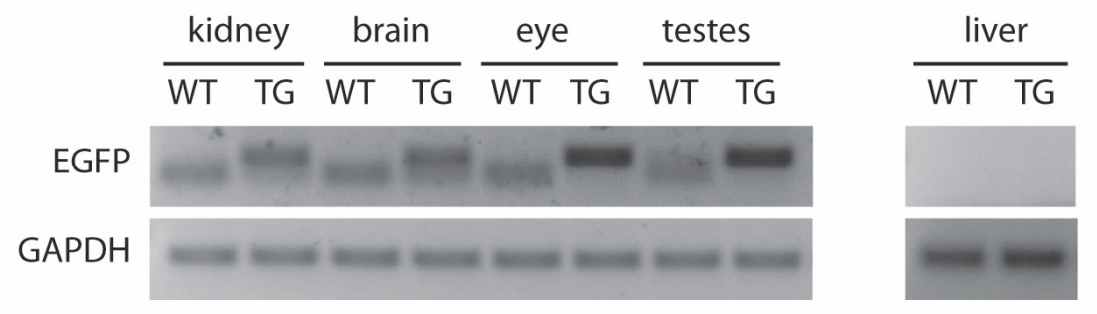

C

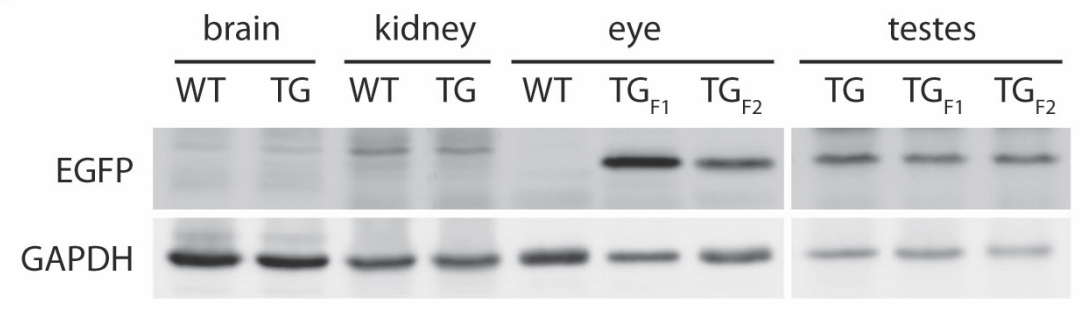

D
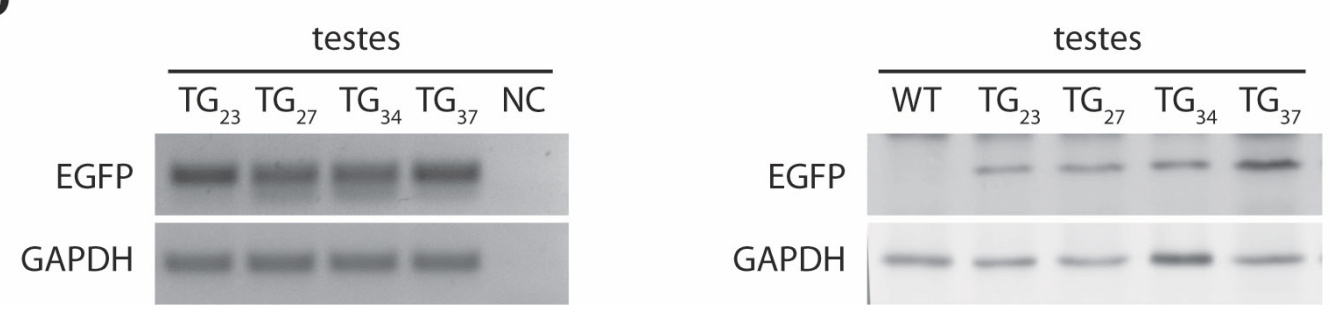

Fig. 1. Generation and analysis of LRAT-EGFP reporter mice. A) The LRAT-EGFP transgene contains truncated LRAT promoter (LRAT1166/+262) and EGFP CDNA followed by the chimeric intron (INT), and a SV40 poly-A signal (SV40(A)) between transposon inverted terminal repeats (IR-L, IR-R). B) Kidney, brain, eye, testes and liver tissue samples from wild-type (WT) and transgene (TG) mice were analyzed for EGFP transcript by semi-quantitative PCR. Note, positive EGFP PCR product corresponds to 700 bp in TG, nonspecific PCR product runs at lower molecular weight in WT. GAPDH, housekeeping control. C) Tissue samples of kidney, brain, eye and testes were analyzed by immunoblotting for EGFP expression. GAPDH, loading control. D) Tissue samples of testes dissected from WT and TG mice, aged $23\left(\mathrm{TG}_{23}\right), 27\left(\mathrm{TG}_{27}\right), 34\left(\mathrm{TG}_{34}\right)$ and $37\left(\mathrm{TG}_{37}\right)$ days, were analyzed for EGFP expression by semi-quantitative PCR (left panel) and immunoblotting (right panel). NC, negative control; GAPDH, housekeeping (left panel) and loading (right panel) control.

\section{Results}

Murine LRAT promoter analysis and generation of LRAT-reporter mouse

In this study we chose proximal LRAT promoter to drive expression of EGFP based on its reported expression in hepatic stellate cells (Nagatsuma et al. 2009). To generate the LRAT-reporter mouse 5'-untranslated region containing altogether $-1428 \mathrm{bp}$ including the putative transcriptional start site and 262 bp downstream sequence was synthesized and cloned into the SB-based pT2/Venus vector between the inverted terminal repeats
(IR) replacing thus the Venus (Mates et al. 2009) (Fig. 1A). Our transgenic C57BL/6N-Tg(LRAT-EGFP) reporter line was generated by pronuclear co-microinjection of the transgenic constructs and transposase SB100 mRNA into zygotes derived from C57BL/6N strain. We obtained seventeen transgenic founders (three males and four females) that showed integration of the transgene. All founders were characterized using semi-quantitative PCR and Western blotting regarding the EGFP expression in organs with expected LRAT expression, i.e. in the liver, kidney, brain, eye, and testes (Fig. 1B-D). None of the founders showed detectable expression in the liver, kidney, 
brain but in eyes and testis (Fig. 1B-D). The F1 and F2 lines were further analyzed using immunostaining with either anti-EGFP antibodies or direct EGFP signals (Figs 2 and 3).

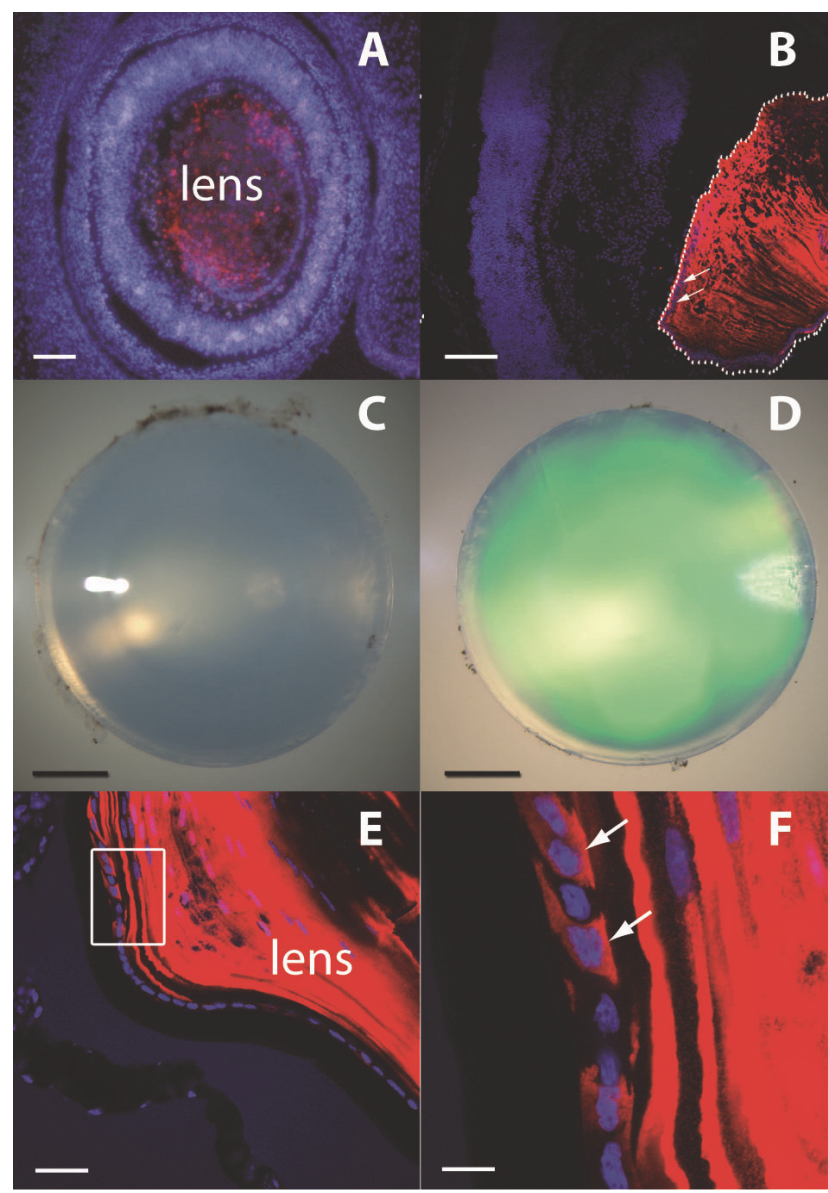

Fig. 2. Expression of EGFP protein in lens and lens epithelium of LRAT-EGFP reporter transgenic mouse from embryogenesis to adulthood. Immunohistochemical detection of EGFP (red) with nuclear counterstain (DAPI, blue). Shown are eyes from representative $14.5 \mathrm{dpc}$ embryo (A), PN1 pup (B) and adult $(\mathbf{E}, \mathbf{F})$. Fresh preparation of adult lens visualized by direct fluorescence microscopy $(\mathbf{C}, \mathbf{D})$. Nuclei were stained with DAPI (blue). Dotted lines in B outline the lens. Scale bar in A, B, E = $100 \mu \mathrm{m} ; \mathrm{C}, \mathrm{D}=1 \mathrm{~mm}$, and $\mathrm{F}=12.5 \mu \mathrm{m}$.

LRAT-EGPP reporter is expressed in eyes from embryonic stage to adulthood

EGFP expression in the eyes was obvious and bright EGFP-signal was directly observable in lenses using fluorescence stereomicroscope (Fig. 2). To characterize the expression at the cellular level, eyes were sectioned and the EGFP expression demonstrated by immunofluorescence. EGFP is observed in the lens of transgenic LRAT-EGFP reporter from 14.5 day post coitus (dpc) to adulthood. At higher magnification, the EGFP expression is not only observed in lens but also in the lens epithelium and lens fibers, all of which are continued to be expressed in adult (Fig. 2A-F).

\section{LRAT-EGFP expression in testis}

LRAT has been reported to be expressed in Sertoli cells and be involved in testis development (Shingleton et al. 1989). Schmitt and Ong (1993) reported LRAT activity in round spermatid-enriched cells, the post meiotic germ cells, from postpubertal rat testis (Moise et al. 2007).

At postnatal day (PN) 7, expression of EGFP in testis of LRAT-EGFP transgenic mice is not significantly above background (not shown). By PN 14, we observed weak EGFP expression in Sertoli cells. These cells form and single cell layer encasing testicular cord, can have distinguished oval shape nucleus often indented and oriented perpendicular to the basal membrane with the strikingly noticeable nucleolus (Zolfaghari and Ross 2009) (arrowhead, Fig. 3A). Interestingly, we observed very strong EGFP expression in both nucleus and cytoplasm specifically in meiotic spermatids and not in spermatogonia. The spermatocytes usually reside further away while the spermatogonia are attached to the seminiferous membrane (arrows, Fig. 3B). This expression is in agreement with the initiation of secondary meiosis in the mouse, which begins around PN10 (Liu and Gudas 2005).

In adult testis, we detected EGFP expression strongly in spermatocytes undergoing meiosis as well as postmeiotic cells in spermatogenesis, which include round spermatids, elongating and elongated spermatids (Fig. 3B, C). EGFP expression in Sertoli cells appears to be reduced and less noticeable compare to EGFP expression in spermatocytes and spermatids (Fig. 3B).

To clarify meiotic spermatocytes in adult testis, we perform co-localization of anti-EGFP and anti-SCP3 antibodies, which stains for synaptonemal complexes protein 3, a bona fide marker of meiosis (Yuan et al. 2000). SCP3 expresses during meiosis in spermatocytes and not in spermatogonia where division is accomplished by mitosis. In the testis, SCP3 begins to express from leptotene spermatocytes to late meiotic cells when the expression ceases. LRAT-EGFP expression was detected in all SCP3 positive cells as well as the SCP3 negative postmeiosis spermatids, judged by the location of these cells near the center of seminiferous tubules. There is no EGFP expression in spermatogonia cells (arrowheads, Fig. 3C), which is in agreement with our data in PN14 aforementioned (Fig. 3C). 


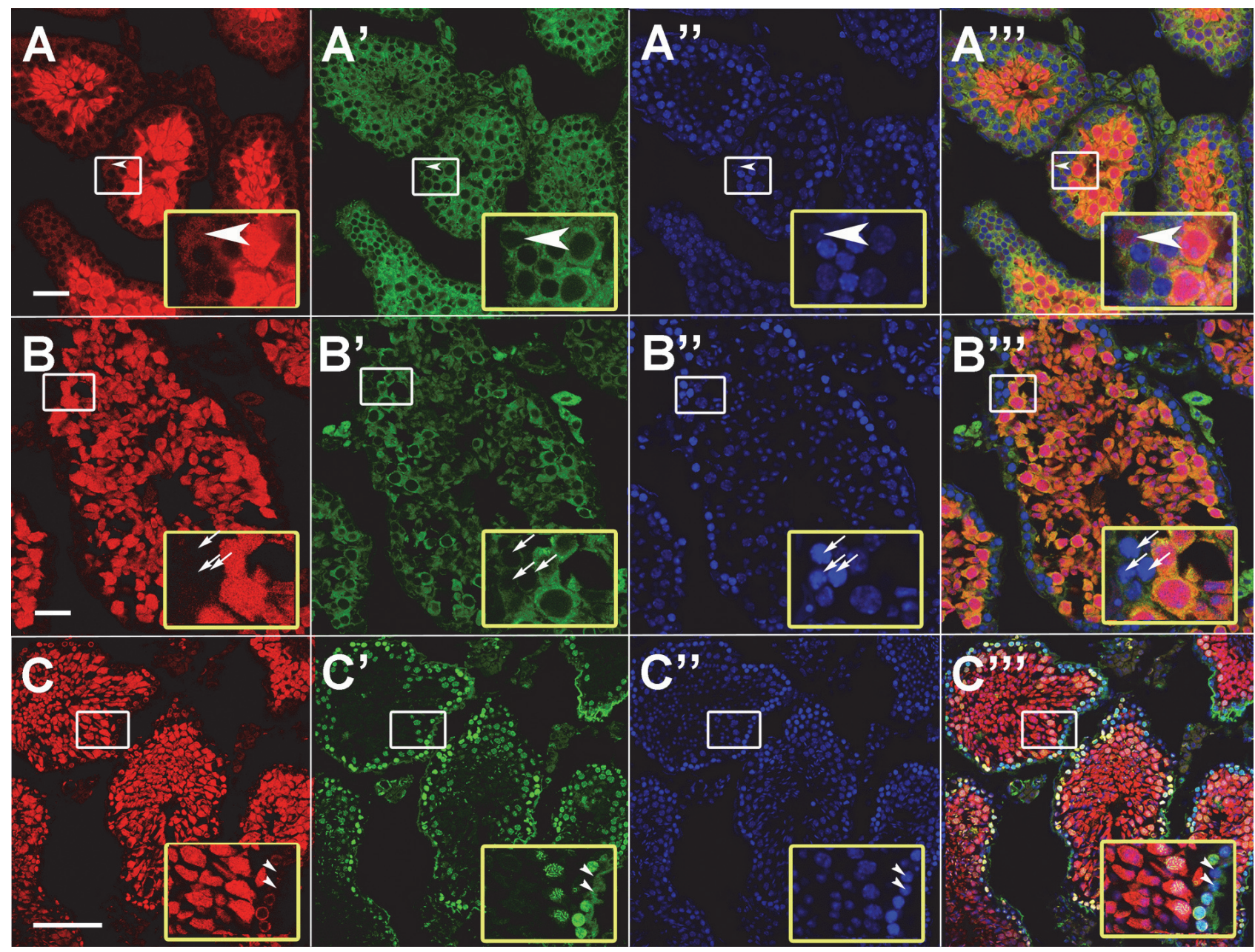

Fig. 3. Expression of EGFP protein in testis of LRAT-EGFP reporter transgenic mouse from postnatal day 14 to adulthood. Immunofluorescence staining of cross sections of tubuli seminiferi: Panel $\mathbf{A}$ is postnatal day 14 , $\mathbf{B}$ and $\mathbf{C}$ are adult. Shown are antiEGFP (red, A, B, C), anti-DDX4 (green, A', B'), anti-SCP3 (green, $\mathbf{C}^{\prime}$ ) and DAPI nuclear staining (blue, A", $\mathbf{B}^{\prime \prime}$ and $\mathbf{C}^{\prime \prime}$ ). A'", $\mathbf{B}^{\prime \prime \prime}$ and $\mathbf{C}^{\prime \prime \prime}$ are composite images of all channels. From postnatal day 14 to adulthood, EGFP protein (red) expression in the LRAT-EGFP reporter mouse is highly expressed in meiosis round spermatocytes (SCP3 positive) and in post meiosis spermatids (SCP3 negative) which are located more luminal. Interestingly, we detected EGFP protein in nuclei of Sertoli cells only in postnatal day 14 (arrowhead in panel A). Scale bar $=100 \mu \mathrm{m}$. Insets show higher magnification view of area delineated by the white rectangles.

\section{Discussion}

LRAT is known to have the predominant enzymatic function in the liver where it is responsible for the formation of retinyl esters and essential for vitamin A storage (Batten et al. 2004, Liu and Gudas 2005, Moise et al. 2007, Zhang et al. 2012). We have generated transgenic mouse lines where EGFP expression is under the control of proximal LRAT promoter $(-1166$ to +262$)$. Analysis of EGFP protein expression by Western blotting and immunostaining showed EGFP expression in subsets of LRAT endogenous expressing domains such as lens and testis but not in retinal epithelium, liver, lung, intestine, where endogenous LRAT is expressed. This expression pattern does not fully recapitulate the described expression of endogenous LRAT gene in spite of the promoter region contained the necessary elements for high activity of luciferase such as TATA box, SP3 site, AP-1 site, and CAAT box, all of which are essential and well-conserved in rat, human, and mouse (Zolfaghari and Ross 2009). The differences in the expression underline the necessity of additional regulatory elements essential to drive complex expression of endogenous LRAT in tissue-specific manner.

In the eye, we showed expression of EGFP in the lens epithelium and the lens fibers which actively differentiated from equatorial zone of lens epithelium throughout life (Bhat 2001) but not in the retinal pigment epithelium (RPE). This finding is different to published reports of endogenous LRAT expression, which showed specific expression in the RPE (Batten et al. 2004). Lack of Lrat function in mice resulted in slightly shortened rod 
outer segment layer in the retina and no defects in any other layer (Batten et al. 2004). We did not detect endogenous LRAT by our immunostaining during embryonic development or in adulthood. Collectively, this suggested that endogenous LRAT is either not expressed and/or dispensable for lens development and differentiation.

In testis, LRAT is well known to be expressed in Sertoli cells but its expression in germ cells has not been described in detail. The EGFP transgene under the control of the proximal Lrat promoter was also expressed in Sertoli cells, however, it was also detected in meiosis spermatocytes as well as in post meiotic cells undergoing spermatogenesis. The latter expression is in agreement with a report identifying the highest LRAT enzymatic activity occurring in microsomal and cytosolic preparations of post meiosis round spermatids as compared to Sertoli cells and other cells types in the developing rat testis (Schmitt and Ong 1993). Furthermore, male mice lacking functional Lrat showed oligospermia but no changes in mRNA expression of genes specific for spermatogenesis compare to wild-type (Liu and Gudas 2005). The authors concluded that lack of Lrat causes an absence in mature sperm and that spermatogenesis is compromised but is not completely disrupted. There was no further characterization of the oligospermia, nonetheless the data confirms the importance of LRAT in sperm formation.

Subsequent studies showed that male Lrat mutants embryos under the influence of a maternal vitamin A-deficient diet from the time of post conception to weaning and thereafter fed the same vitamin A deficient diet fail to undergo postnatal meiosis and remain as undifferentiated spermatogonias (Li et al. 2011). Our LRAT-EGFP transgenic reporter mice exhibit expression of EGFP in meiosis spermatocyte and post meiosis spermatids and not in spermatogonia from PN14. This transgenic mouse line could be used as a negative marker to prepare spermatogonia (EGFP negative) population in isolating spermatogonial stem cells from adult mouse testis (Guan et al. 2006).

In summary, our transgenic mouse line expressing EGFP under the proximal LRAT promoter showed expression in the eyes and testis. This expression does not fully recapitulate reported endogenous Lrat gene expression, underscoring the requirement of additional regulatory elements and/or enhancers necessary to govern complex expression of endogenous LRAT. Nevertheless, this LRAT-transgenic reporter mouse might be useful to further characterize spermatogenesis.

\section{Conflict of Interest}

There is no conflict of interest.

\section{Acknowledgements}

We thank L. Sarnova and V. Mihalova for their outstanding technical assistance. We are also grateful to Dr. T. Epp and K. Chawengsaksophak for critical reading of the manuscript. Financial support was given to RS by GACR (P305/10/2143), Czech Academy of Sciences (RVO 68378050), and by Ministry of Education, Youth and Sports, Czech Republic (OP RDI CZ.1.05/1.1.00/02.0109 "Biotechnology and Biomedicine Centre of the Czech Academy of Sciences and Charles University in Vestec (BIOCEV)").

\section{References}

BATTEN ML, IMANISHI Y, MAEDA T, TU DC, MOISE AR, BRONSON D, POSSIN D, VAN GELDER RN, BAEHR W, PALCZEWSKI K: Lecithin-retinol acyltransferase is essential for accumulation of all-transretinyl esters in the eye and in the liver. J Biol Chem 279: 10422-10432, 2004.

BHAT SP: The ocular lens epithelium. Biosci Rep 21: 537-563, 2001.

D'AMBROSIO DN, CLUGSTON RD, BLANER WS: Vitamin A metabolism: an update. Nutrients 3: 63-103, 2011.

GUAN K, NAYERNIA K, MAIER LS, WAGNER S, DRESSEL R, LEE JH, NOLTE J, WOLF F, LI M, ENGEL W, HASENFUSS G: Pluripotency of spermatogonial stem cells from adult mouse testis. Nature 440: 1199-1203, 2006.

GUO X, KNUDSEN BS, PEEHL DM, RUIZ A, BOK D, RANDO RR, RHIM JS, NANUS DM, GUDAS LJ: Retinol metabolism and lecithin:retinol acyltransferase levels are reduced in cultured human prostate cancer cells and tissue specimens. Cancer Res 62: 1654-1661, 2002.

HOGARTH CA, GRISWOLD MD: The key role of vitamin A in spermatogenesis. J Clin Invest 120: 956-962, 2010. 
JOHNSON PM, CLIFT LE, ANDRLIKOVA P, JURSOVA M, FLANAGAN BF, CUMMERSON JA, STOPKA P, DVORAKOVA-HORTOVA K: Rapid sperm acrosome reaction in the absence of acrosomal CD46 expression in promiscuous field mice (Apodemus). Reproduction 134: 739-747, 2007.

KAWAGUCHI R, YU J, HONDA J, HU J, WHITELEGGE J, PING P, WIITA P, BOK D, SUN H: A membrane receptor for retinol binding protein mediates cellular uptake of vitamin A. Science 315: 820-825, 2007.

LI H, PALCZEWSKI K, BAEHR W, CLAGETT-DAME M: Vitamin A deficiency results in meiotic failure and accumulation of undifferentiated spermatogonia in prepubertal mouse testis. Biol Reprod 84: 336-341, 2011.

LIU L, GUDAS LJ: Disruption of the lecithin:retinol acyltransferase gene makes mice more susceptible to vitamin A deficiency. J Biol Chem 280: 40226-40234, 2005.

LIU L, TANG XH, GUDAS LJ: Homeostasis of retinol in lecithin: retinol acyltransferase gene knockout mice fed a high retinol diet. Biochem Pharmacol 75: 2316-2324, 2008.

MATES L, CHUAH MK, BELAY E, JERCHOW B, MANOJ N, ACOSTA-SANCHEZ A, GRZELA DP, SCHMITT A, BECKER K, MATRAI J, MA L, SAMARA-KUKO E, GYSEMANS C, PRYPUTNIEWICZ D, MISKEY C, FLETCHER B, VANDENDRIESSCHE T, IVICS Z, IZSVAK Z: Molecular evolution of a novel hyperactive Sleeping Beauty transposase enables robust stable gene transfer in vertebrates. Nat Genet 41: 753-761, 2009.

MCBEE JK, PALCZEWSKI K, BAEHR W, PEPPERBERG DR: Confronting complexity: the interlink of phototransduction and retinoid metabolism in the vertebrate retina. Prog Retin Eye Res 20: 469-529, 2001.

MOISE AR, NOY N, PALCZEWSKI K, BLANER WS: Delivery of retinoid-based therapies to target tissues. Biochemistry 46: 4449-4458, 2007.

O'BYRNE SM, BLANER WS: Retinol and retinyl esters: biochemistry and physiology. J Lipid Res 54: 1731-1743, 2013.

PAIK J, VOGEL S, QUADRO L, PIANTEDOSI R, GOTTESMAN M, LAI K, HAMBERGER L, VIEIRA MDE M, BLANER WS: Vitamin A: overlapping delivery pathways to tissues from the circulation. $J$ Nutr 134: 276S280S, 2004.

PERUSEK L, MAEDA T: Vitamin A derivatives as treatment options for retinal degenerative diseases. Nutrients 5: 2646-2666, 2013.

RUIZ A, GHYSELINCK NB, MATA N, NUSINOWITZ S, LLOYD M, DENNEFELD C, CHAMBON P, BOK D: Somatic ablation of the Lrat gene in the mouse retinal pigment epithelium drastically reduces its retinoid storage. Invest Ophthalmol Vis Sci 48: 5377-5387, 2007.

SAARI JC, BREDBERG DL, FARRELL DF: Retinol esterification in bovine retinal pigment epithelium: reversibility of lecithin:retinol acyltransferase. Biochem J 291: 697-700, 1993.

SCHMITT MC, ONG DE: Expression of cellular retinol-binding protein and lecithin-retinol acyltransferase in developing rat testis. Biol Reprod 49: 972-979, 1993.

SHINGLETON JL, SKINNER MK, ONG DE: Retinol esterification in Sertoli cells by lecithin-retinol acyltransferase. Biochemistry 28: 9647-9653, 1989.

WALD G: Molecular basis of visual excitation. Science 162: 230-239, 1968.

YUAN L, LIU JG, ZHAO J, BRUNDELL E, DANEHOLT B, HOOG C: The murine SCP3 gene is required for synaptonemal complex assembly, chromosome synapsis, and male fertility. Mol Cell 5: 73-83, 2000.

ZHANG T, BAEHR W, FU Y: Chemical chaperone TUDCA preserves cone photoreceptors in a mouse model of Leber congenital amaurosis. Invest Ophthalmol Vis Sci 53: 3349-3356, 2012.

ZOLFAGHARI R, ROSS AC: An essential set of basic DNA response elements is required for receptor-dependent transcription of the lecithin:retinol acyltransferase (Lrat) gene. Arch Biochem Biophys 489: 1-9, 2009. 